The Astrophysical JournaL, 443:265-270, 1995 April 10

(C) 1995. The American Astronomical Society. All rights reserved. Printed in U.S.A.

\title{
MUCH ADO ABOUT NOTHING: SEVERAL LARGE-AREA SURVEYS FOR RADIO PULSARS FROM ARECIBO
}

\author{
Paul S. Ray, ${ }^{1}$ W. T. S. Deich, S. R. Kulkarni, Thomas A. Prince, \\ S. E. Thorsett, ${ }^{2}$ AND Gautam Vasisht \\ Division of Physics, Mathematics and Astronomy, California Institute of Technology, Pasadena, CA 91125 \\ A. WolszCZan \\ Department of Astronomy and Astrophysics, Pennsylvania State University, University Park, PA 16802 \\ Donald C. Backer and John H. Holliman II \\ Radio Astronomy Laboratory, University of California, Berkeley, CA 94720 \\ AND \\ JOHN MidDLEDITCH \\ Los Alamos National Laboratory MS B265 C-3, Los Alamos, NM 87545 \\ Received 1994 August 19 ; accepted 1994 October 20
}

\begin{abstract}
We report surveyed regions and flux limits for several large-area surveys at $430 \mathrm{MHz}$ for radio pulsars using the $305 \mathrm{~m}$ reflector at Arecibo, Puerto Rico. The surveys used integration times between 8 and $67 \mathrm{~s}$ and covered a total area of $\sim 515$ square degrees. No previously undiscovered pulsars were detected in these regions, although all previously known pulsars were detected. The nondetection of new pulsars places constraints on the population of low-luminosity pulsars and must be taken into account in estimates of the local density of millisecond pulsars.
\end{abstract}

Subject headings: pulsars: general — radio continuum: stars — stars: statistics — surveys

\section{INTRODUCTION}

The $305 \mathrm{~m}$ radio telescope at Arecibo, Puerto Rico, can observe a strip of sky from declinations $-1^{\circ} 39^{\prime}$ to $+38^{\circ} 21^{\prime}-$ over 13,000 square degrees. However, before 1990 virtually all of the untargeted pulsar searching from Arecibo was confined to a narrow strip of less than $10^{\circ}$ around the Galactic plane, and toward the Galactic center (Hulse \& Taylor 1974; Damashek, Taylor, \& Hulse 1978; Stokes et al. 1986; Nice 1992). This observing strategy maximizes the number of pulsars discovered, since pulsars are clustered toward the Galactic plane. As a consequence, the pulsar distribution is well measured near the plane, but substantially less constrained at high Galactic latitudes. Complete surveys of high latitudes, while they discover fewer pulsars per square degree, are important because discovery of low-luminosity (nearby) pulsars is equally likely at high latitudes, and measuring the low-luminosity cutoff of the pulsar luminosity function is important for determination of the total pulsar population and birth rate. In addition, observations of high-latitude pulsars can help map the interstellar electron distribution in our local region.

The early surveys also had sample times much too long to be sensitive to millisecond pulsars, which provide the most interesting astrophysical laboratories for binary orbital evolution, time-keeping metrology, discovery of planetary systems, highprecision astrometry, and many other applications. The observed population of millisecond pulsars has a characteristic distance from the Galactic plane of $\gtrsim 600$ pc (Phinney \& Kulkarni 1994), which is similar to that of the low-mass X-ray binary systems that are commonly assumed to be their pro-

\footnotetext{
1 paulr@ccsf.caltech.edu.

${ }^{2}$ Current address: Joseph Henry Laboratories and Department of Physics, Princeton University, Princeton, NJ 08544.
}

genitors. The actual scale height of the millisecond pulsar distribution is probably even larger because of their large ages $\left(\sim 10^{9} \mathrm{yr}\right)$, but selection effects of surveys limit the sample to nearby pulsars. Most millisecond pulsars have been found at distances of less than $3 \mathrm{kpc}$, so their distribution on the sky is expected to be fairly isotropic. The fact that the first two millisecond pulsars were found in the Galactic plane is simply an observational selection effect (Srinivasan \& Bhattacharya 1989). Thus, the known population of millisecond pulsars may be greatly increased by sensitive high-latitude surveys. All but two millisecond pulsars known in the Galactic disk (PSR $\mathrm{J} 2322+2057$ and PSR B1937+21) are in binary systems. For surveys requiring long integration times, this acts as a strong selection effect against short-period binary pulsars because the Doppler-shifted signal does not remain coherent across the observation. However, the Arecibo instrument can detect pulsars as faint as $\sim 1 \mathrm{mJy}$ in a drift scan that integrates $\sim 32 \mathrm{~s}$ (see sensitivity discussion in $\S 4$ ) -a time shorter than the timescale for orbital acceleration to degrade the coherence of the signal. Surveys at smaller telescopes require longer integrations, imposing a large selection effect against short-period binary systems which is not present in the surveys described here.

Hulse \& Taylor (1974) surveyed 24 square degrees toward the Galactic anticenter, but the Nyquist limit of their search was $33.4 \mathrm{~ms}$. Young pulsars found in the direction of the Galactic anticenter could be of great interest. The sky is much less crowded in the anticenter direction than in other regions of the Galactic plane, and hence a young pulsar could be convincingly associated with the molecular cloud from which its parent massive star collapsed. In addition, timable millisecond pulsars at many separate locations are needed to become part of a millisecond pulsar timing array, which has the potential to discover cosmological gravitational waves with periods from 
days to years. For these reasons, we undertook a survey for fast pulsars in the Galactic anticenter.

Then, during 1990 February, while the Arecibo telescope azimuth drive was unusable, A. Wolszczan used the telescope in drift mode to survey $\sim 150$ square degrees of sky. Two interesting pulsars (PSR B1257+12, a millisecond pulsar with a planetary system, and PSR B $1534+12$, a relativistic binary system) were discovered in this survey (Wolszczan 1990, 1991; Wolszczan \& Frail 1992). Spurred by this success, the anticenter survey was enlarged, and several groups began an effort to survey the entire Arecibo sky for millisecond pulsars. This is continuing during the current upgrade of the Arecibo telescope. Also, the two pulsars discovered by Wolszczan, using $32 \mathrm{~s}$ integrations, had flux densities much higher than his survey limit. This motivated us to undertake a survey with a much shorter integration time, $8.2 \mathrm{~s}$ per beam.

We report on several major pulsar searches carried out at the Arecibo Observatory from 1988 to 1993. All surveys covered large areas of sky that had not been previously searched with comparable sensitivity or sampling rates required to detect millisecond pulsars.

In $\S 2$ we describe the hardware, observation strategy, and pulsar detections for each of the surveys. In $\S 3$ we describe the data analysis techniques. In $\S 4$ we discuss the sensitivity of the surveys and implications for the Galactic population of millisecond pulsars, and the low-luminosity cutoff of the normal pulsar population.

\section{SURVEYS}

All surveys used the same telescope, radio frequency, receivers, and analysis software. The survey parameters are summarized in Table 1 . They differed in sky coverage, pointing strategy, integration time, and spectrometer.

All surveys employed the $430 \mathrm{MHz}$ line feed at the Arecibo $305 \mathrm{~m}$ radio telescope. The feed received two circular polarizations using cryogenically cooled receivers in the carriage house. The feed/receiver system has an inherent half-power bandpass of $10.2 \mathrm{MHz}$ (National Astronomy and Ionosphere Center 1989, hereafter NAIC) and a forward gain at zenith of $18 \mathrm{~K}$ $\mathrm{Jy}^{-1}$. Typically, the center frequency of the observation was set to $428.5 \mathrm{MHz}$, near the peak sensitivity of the receiver system, but sometimes values of up to $430 \mathrm{MHz}$ were used. The halfpower beam width of the $430 \mathrm{MHz}$ observing system is $10^{\prime}$.

Observations at high Galactic latitudes employed a $2 \times 32 \times 0.25 \mathrm{MHz}$ filterbank, covering a total of $8 \mathrm{MHz}$ in bandwidth for each polarization. The signal in each channel was square-law detected and smoothed with a time constant of $330 \mu \mathrm{s}$. The two polarizations were then summed and the result digitized with 3 bit precision. Sample times for the digitizer were between 180 and $250 \mu \mathrm{s}$. Because of the relatively wide channel bandwidths, the filter bank induces significant loss of sensitivity to $1.0 \mathrm{~ms}$ pulsars at a dispersion measure (DM) of $\sim 20 \mathrm{pc} \mathrm{cm}^{-3}$ (the dispersion-induced smearing across one filter bank channel is $260 \mu \mathrm{s} \times \mathrm{DM} / 10 \mathrm{pc} \mathrm{cm}^{-3}$ ).

The anticenter (AC) survey ( $\$ 2.3$ ) used the Arecibo autocorrelation spectrometer (NAIC 1989), which can sample only as fast as $\sim 500 \mu \mathrm{s}$, but which has a $10 \mathrm{MHz}$ bandwidth and much narrower channels $(78.125 \mathrm{kHz}$, as compared to $250 \mathrm{kHz})$, allowing searches at DMs up to $60 \mathrm{pc} \mathrm{cm}^{-3}$ with negligible loss of sensitivity from dispersion smearing. The correlator divided the band into four $2.5 \mathrm{MHz}$ banks, each of which is fed into a 32 lag autocorrelator. Each correlator integrated for 500 $\mu$ s and then was inactive for $6 \mu$ s while it dumped its data to the control computer. There was an additional dead time of $40 \mu \mathrm{s}$ when each block of 64 samples was dumped, resulting in an average time between samples of $506.625 \mu \mathrm{s}$. These autocorrelation functions for each polarization along with additional 12 bit total power counters were blocked into records and written to magnetic tape by the Harris $\mathrm{H} 800$ control computer.

\subsection{Slewing and Drifting High-Latitude Surveys (SHL and DHL)}

In this survey, observations were made while slewing the telescope, hence the designation as the Slewing High-Latitude (or SHL) survey. Sky coverage was between Galactic latitudes $-15^{\circ}$ and $-40^{\circ}$. At these high latitudes, the expected maximum DM is only $\sim 30 \mathrm{pc} \mathrm{cm}^{-3}$ (Taylor \& Cordes 1993), which yields a smearing of $750 \mu$ s across one of the $250 \mathrm{kHz}$ channels. Thus, the benefits of faster sampling and somewhat simpler data reduction made the filter bank appropriate for this project.

The Arecibo telescope can point only within $20^{\circ}$ of the zenith, and the sensitivity decreases and system temperature increases as the pointing moves away from the zenith. Therefore, maximum sensitivity is obtained when objects are observed at transit. For this reason, observations began at zenith and the telescope was driven at $1 \mathrm{hr}$ of right ascension (R.A.) per hour of time. To achieve faster sky coverage, the telescope was driven north at $70^{\circ} 30^{\prime} \mathrm{hr}^{-1}$ for 4 minutes and then reversed for 4 minutes; this cycle was repeated through-

TABLE 1

Summary of Surveys Included in This PaPer

\begin{tabular}{|c|c|c|c|c|}
\hline Parameter & $\mathrm{AC}$ & SHL & DHL & CBHL \\
\hline Backend $\ldots \ldots \ldots \ldots \ldots \ldots \ldots \ldots \ldots \ldots \ldots \ldots$ & Autocorrelator & Filter bank & Filter bank & Filter bank \\
\hline Sample interval $(\mu \mathrm{s}) \ldots \ldots \ldots \ldots \ldots$ & 506.625 & 250 & 250 & 180 \\
\hline Integration time (s) ................. & 67,32 & 8 & 32 & 47,23 \\
\hline Observing frequency $(\mathrm{MHz}) . . . \ldots$. & 430 & 430 & 430 & 428.5 \\
\hline Total bandwidth $(\mathrm{MHz})$........... & 10 & 8 & 8 & 8 \\
\hline Channel bandwidth $(\mathrm{kHz})$.......... & 78.125 & 250 & 250 & 250 \\
\hline RFI-free area (square degree)...... & $100^{\mathrm{a}}$ & 285 & 63 & 68 \\
\hline R.A. range $\ldots \ldots \ldots \ldots \ldots \ldots \ldots$ & $04^{\mathrm{h}}$ to $09^{\mathrm{h}}$ & $01^{\mathrm{h}}$ to $04^{\mathrm{h}}$ & $01^{\mathrm{h}}$ to $04^{\mathrm{h}}$ & $08^{\mathrm{h}}$ to $13^{\mathrm{h}}$ \\
\hline Decl. range $\ldots \ldots \ldots \ldots \ldots \ldots \ldots \ldots$ & $+11^{\circ}$ to $+25^{\circ}$ & $+22^{\circ}$ to $+35^{\circ}$ & $+19^{\circ} .5$ to $+22^{\circ}$ & $+13^{\circ}$ to $+18^{\circ}$ \\
\hline 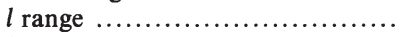 & $170^{\circ}$ to $210^{\circ}$ & $125^{\circ}$ to $170^{\circ}$ & $126^{\circ}$ to $170^{\circ}$ & $205^{\circ}$ to $319^{\circ}$ \\
\hline$b$ range $\ldots \ldots \ldots \ldots \ldots \ldots \ldots \ldots \ldots \ldots \ldots \ldots \ldots$ & $-30^{\circ}$ to $+30^{\circ}$ & $-13^{\circ}$ to $-40^{\circ}$ & $-22^{\circ}$ to $-43^{\circ}$ & $+21^{\circ}$ to $+75^{\circ}$ \\
\hline
\end{tabular}

NoTES.-Telescope diameter (m): 305; Number of polarizations: 2 ; Receiver gain: $18 \mathrm{~K} \mathrm{Jy}^{-1}$; Noise temperature: $90 \mathrm{~K}$.

Approximate. 
out the observation. This pointing scheme traced a zig-zag pattern on the sky. For the next observation, the entire pattern was shifted in R.A. by a beam width. If this is completed for all starting positions, then a strip of sky of height $04^{\circ} 42^{\prime}$ is covered twice (once by a northwest-slewing scan and once by a southward-slewing scan). Although the survey proceeds only twice as fast as a traditional drift survey, the double coverage is advantageous because both radio-frequency interference (RFI) and pulsar scintillation can cause an otherwise detectable pulsar to be missed on the first pass.

The RFI-free sky coverage of this survey is included in Figure 1. The survey covers the region $1^{\mathrm{h}}<\alpha<04^{\mathrm{h}}$, $22^{\circ}<\delta<35^{\circ}$ with a filling factor of $\sim 50 \%$, for a total of 285 square degrees. About 105 square degrees of this region were covered more than once by interference-free observations. There are no known pulsars in the survey region. These data were taken during two observing sessions: 1991 September 8-18 and 1991 October 13-November 3. This survey has a minimum detectable flux twice that of a normal drift survey for fast pulsars, but the limit is somewhat worse for slow pulsars. This is predominantly due to a very large red-noise component in the power spectra generated by sky background changes as the telescope beam slewed across the sky. For drift surveys, the variation is slow and is removed by $\mathrm{AC}$ coupling the postdetection signal with a time constant of $\sim 12 \mathrm{~s}$. This time constant was too long for the slewing survey, and it produced power spectra that were difficult to search for slow pulsars. The sensitivity of this survey is discussed further in $\S 4$.

No pulsars were detected in the 1991 September-October data. At that time, other surveys (Nice 1992; Camilo, Nice, \& Taylor 1993; Foster, Wolszczan, \& Camilo 1993) were successfully discovering millisecond pulsars which were much nearer the detection limit of drift surveys. Since similar pulsars might have been missed by the fast slewing survey, the Drifting High Latitude (DHL) survey in 1993 February 1-14 used the

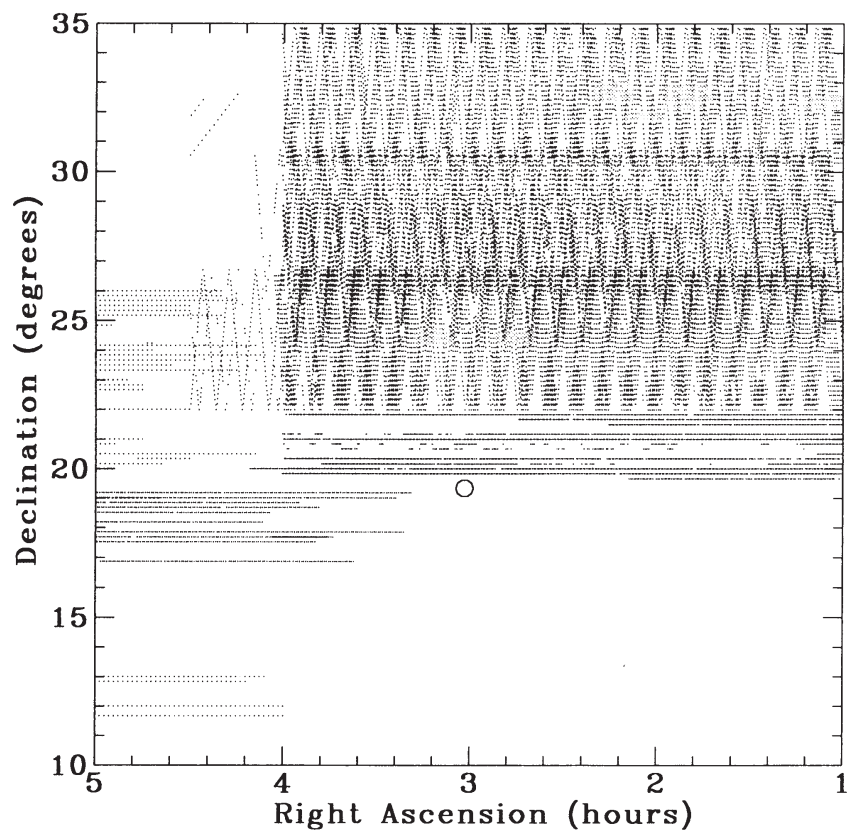

FIG. 1.-RFI-free sky coverage (B1950) of these surveys from $1^{\text {h }}$ to $5^{\text {h }}$ right ascension. The drift scans are separated in declination by $10^{\prime}$. Gaps in the scans are from sporadic RFI or observing problems. Filled circles represent known pulsars detected in these surveys, while open circles are other known pulsars. drift survey mode, with $32 \mathrm{~s}$ integrations. No pulsars were detected in these data. The sky coverage was $01^{\mathrm{h}}<\alpha<04^{\mathrm{h}}$ and $19^{\circ} 40^{\prime}<\delta<21^{\circ} 50^{\prime}$. The RFI-free sky coverage, which totals $\sim 63$ square degrees, is shown on Figure 1.

\subsection{The Caltech-Berkeley High Latitude Survey (CBHL)}

This survey used the filter bank. The data were acquired between 1991 September and 1993 May, in the region $8^{\text {h }}<\alpha<13^{\text {h }}$ and $12^{\circ}<\delta<18^{\circ}$. The sky coverage that was unaffected by RFI totaled 68 square degrees. Of this total, 27 square degrees were surveyed as the sky drifted past the telescope beam at the sidereal rate, giving an effective integration time of $\sim 42 \mathrm{~s}$ per point. The resulting search sensitivity as a function of pulsar period and dispersion measure is identical to Figure 2 of Thorsett et al. (1993). The remaining 41 square degrees were searched with the telescope slewing east at the sidereal rate along lines of contstant declination, giving an effective integration time per point of $21 \mathrm{~s}$. The sensitivity in this part of the sky was a factor $2^{1 / 2}$ worse than that in the first region.

Because of a scheduling error, a large fraction of this survey was directed at regions of the sky already surveyed by Wolszczan (1990). Thus, it is not surprising that no new pulsars were found, though both known pulsars in the search region were detected, including the millisecond pulsar PSR B1257+12. The sky coverage of this survey is shown on Figures 2 and 3.

\subsection{Galactic Anticenter $(A C)$ Survey}

This survey consisted of three observing sessions: 1988 November-1989 January, 1989 November-December, and 1991 January-February. The first two sessions used 67-second pointings on a grid with 22.5 spacing, while the final session used the drift scan method. A total of $\sim 100$ square degrees of RFI-free sky were searched and analyzed (see Figs. 1 and 2). The survey was primarily in the region with $170^{\circ}<l<210^{\circ}$,

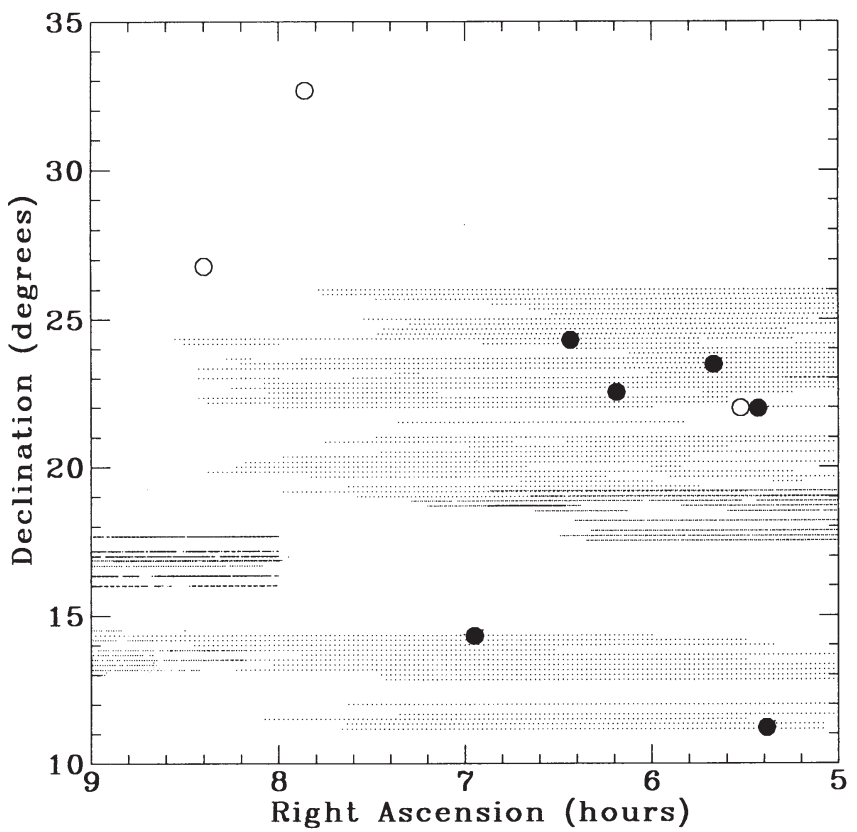

FIG. 2.-RFI-free sky coverage (B1950) of these surveys from $5^{\mathrm{h}}$ to $9^{\mathrm{h}}$ right ascension. The drift scans are separated in declination by $10^{\prime}$. Gaps in the scans are from sporadic RFI or observing problems. Filled circles represent known pulsars detected in these surveys, while open circles are other known pulsars. 


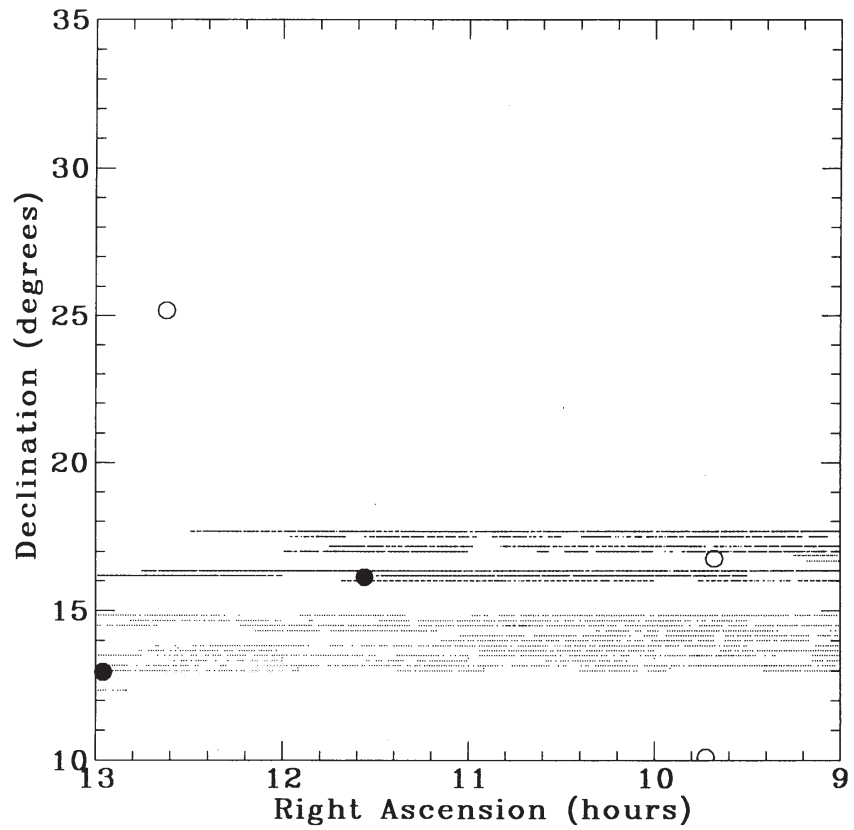

FIG. 3.-RFI-free sky coverage (B1950) of these surveys from $9^{\mathrm{h}}$ to $13^{\mathrm{h}}$ right ascension. The drift scans are separated in declination by $10^{\prime}$. Gaps in the scans are from sporadic RFI or observing problems. Filled circles represent known pulsars detected in these surveys, while open circles are other known pulsars.

and with $|b|<20^{\circ}$. All six known pulsars within the survey region were detected.

In the anticenter direction, the expected DM at $2 \mathrm{kpc}$ is 55 $\mathrm{pc} \mathrm{cm}{ }^{-3}$ and at $10 \mathrm{kpc}$ is $155 \mathrm{pc} \mathrm{cm}^{-3}$ (Taylor \& Cordes 1993). The correlator was used because these DMs result in an unacceptable loss in sensitivity to fast pulsars due to pulse smearing within one of the filter bank channels. The survey data were searched up to $\mathrm{DM} \sim 300 \mathrm{pc} \mathrm{cm}^{-3}$.

\section{DATA ANALYSIS}

The data analysis for the nonpointed surveys (all but the AC survey) was performed with groups of up to 12 Sun, IBM, and HP workstations. The suite of programs used for the analysis (PSRPACK) is described by Deich (1994). The $8 \mathrm{~mm}$ tape containing each data segment was mounted on the manager workstation, which ran a script that sequentially read blocks of raw data off the tape $(32,128$, or 256 kilopoints, depending on sampling rate and telescope slew rate). The manager workstation dynamically maximized the work load on the analysis machines by providing each with a new pointing to reduce as soon as it had completed the previous one. To maintain a consistent sensitivity limit along the drift scans, each tape was run through the system twice, offset by one-half of a beam size.

The data reduction script first reads the raw filter bank data, dedisperses it at typically 50 trial DMs, and writes the dedispersed data to a temporary file. The zero-DM trial is searched for sections of the time series that are substantially different from the mean. The anomalous sections, which are usually due to sporadic radar, lightning, or other interference, are replaced by the local median. This file is then subjected to the standard pulsar search scheme of Fourier transforming, generating a power spectrum, and normalizing the power spectrum to its local mean value. Frequencies that correspond to common RFI peaks are then excised from the power spectrum. This spectrum is searched both for individual peaks and for harmonic sums of 2, 3, 4, 8, and 16 frequencies. Candidates are ranked and the best from each of several DM ranges is used to produce a light curve from the dedispersed data file. The candidate list and light curves are then copied to a storage directory for manual sorting later.

The reduced data are later sorted into clean and RFIpolluted catagories. Data are deemed RFI-polluted if the nonzero DM trials cannot be cleaned of spurious signals by both time- and frequency-domain RFI removal techniques. The files of clean data are used to produce maps of RFI-free coverage and to determine positions and parameters of candidates for follow-up. All known pulsars that are in the surveyed area were detected with very high signal-to-noise ratios by these searches; their positions, periods, DMs, fluxes $\left(S_{400}\right)$ and duty cycles $(w / P)$ are reported in Table 2 .

The analysis of the AC survey data proceeded similarly using supercomputers at the Cornell Supercomputing Center, the San Diego Supercomputer Center, and the Jet Propulsion Laboratory. An additional step required in analyzing the data from the correlator is to perform a Fourier transform on the autocorrelation functions to produce power spectra. This step requires four 32 point Fourier transforms per sample.

\section{DISCUSSION}

The minimum detectable flux in a pulsar survey (Dewey 1984; Biggs \& Lyne 1992; Stokes et al. 1986) is

$$
S_{\min }=K\left[\frac{T_{\mathrm{rec}}+T_{\mathrm{sky}}(l, b)+T_{\mathrm{sp}}(\mathrm{ZA})}{G\left(N_{p} B t\right)^{1 / 2}}\right]\left(\frac{w_{\mathrm{eff}}}{P-w_{\mathrm{eff}}}\right)^{1 / 2},
$$

where the factor $K \sim 12$ is empirically determined from detection of known pulsars and expresses the analysis detection threshold $(\sim 8 \sigma) . T_{\text {rec }}, T_{\text {sky }}(l, b)$, and $T_{\text {sp }}(\mathrm{ZA})$ are the receiver temperature, sky temperature (depending on Galactic longitude and latitude), and effective spillover temperature (depending on zenith angle), respectively. Here, $G$ is the tele-

TABLE 2

Previously Known Pulsars Detected in These Surveys

\begin{tabular}{|c|c|c|c|c|c|c|}
\hline Name & $\underset{(\mathrm{J} 2000.0)}{\text { R.A. }}$ & $\begin{array}{c}\text { Decl. } \\
(\mathrm{J} 2000.0)\end{array}$ & $\begin{array}{l}\text { Period } \\
\text { (s) }\end{array}$ & $\begin{array}{c}\mathrm{DM} \\
\left(\mathrm{pc} \mathrm{cm}^{-3}\right)\end{array}$ & $\begin{array}{l}S_{400} \\
(\mathrm{mJy})\end{array}$ & $\begin{array}{l}w / P \\
(\%)\end{array}$ \\
\hline B $0523+11 \ldots \ldots$ & $05^{\mathrm{h}} 25^{\mathrm{m}} 56^{\mathrm{s}} 4$ & $+11^{\circ} 15^{\prime} 19^{\prime \prime}$ & 0.35443 & 79 & 19 & 4.5 \\
\hline $\mathrm{B} 0525+21 \ldots \ldots$ & $\begin{array}{lll}05 & 28 & 52.3\end{array}$ & +220001 & 3.74552 & 51 & 60 & 4.8 \\
\hline B $0540+23 \ldots \ldots$ & $\begin{array}{lll}05 & 43 & 09.6\end{array}$ & +232906 & 0.24596 & 78 & 30 & 2.4 \\
\hline B $0611+22 \ldots \ldots$ & $\begin{array}{lll}06 & 14 & 17.1\end{array}$ & +222958 & 0.33492 & 97 & 30 & 1.9 \\
\hline B $0626+24 \ldots \ldots$ & $\begin{array}{lll}06 & 29 & 05.7\end{array}$ & +241542 & 0.47662 & 84 & 30 & 2.5 \\
\hline B $0656+14 \ldots \ldots$ & $\begin{array}{lll}06 & 59 & 48.1\end{array}$ & +141422 & 0.38487 & 14 & 6 & 3.9 \\
\hline $\mathrm{B} 1133+16 \ldots \ldots$ & $\begin{array}{lll}11 & 36 & 03.0\end{array}$ & +155118 & 1.18791 & 5 & 300 & 2.3 \\
\hline $\mathrm{B} 1257+12 \ldots \ldots$ & $\begin{array}{lll}13 & 00 & 02.7\end{array}$ & +124059 & 0.00622 & 10 & 20 & 9.3 \\
\hline
\end{tabular}


scope gain in units of $\mathrm{K} \mathrm{Jy}^{-1}, N_{p}$ is the number of polarizations received, $B$ is the total bandwidth in $\mathrm{MHz}$, and $t$ is the duration of the observation in seconds. The rightmost factor in equation (1) gives the dependence on the pulse duty cycle, where $P$ is the pulse period, and $w_{\text {eff }}$ is the effective pulse width:

$$
w_{\text {eff }}=\left[w^{2}+\beta^{2} \tau^{2}+\left(\frac{\tau \mathrm{DM}}{\mathrm{DM}_{0}}\right)^{2}\right]^{1 / 2} .
$$

Here, $w$ is the intrinsic pulse width, $\tau$ is the sampling interval in seconds, $\beta \simeq 2$ is related to the time constant of the postdetection smoothing filter, and $\mathrm{DM}_{0}$ is the dispersion measure at which the pulse delay across the bandpass of a single narrowfrequency channel equals the sampling interval. This latter value is

$$
\mathrm{DM}_{0}=1.2 \times 10^{-4} \frac{\tau v^{3}}{\Delta v} \mathrm{pc} \mathrm{cm}^{-3}
$$

where $v$ and $\Delta v$ are the observing frequency and channel bandwidth in $\mathrm{MHz}$, respectively.

Figure 4 shows the flux limit as a function of period for a typical drift survey at Arecibo (e.g., DHL, CBHL) that employs the filter bank. Figure 5 provides the same information for the autocorrelator. Pulsars detected in the surveys are plotted with their cataloged flux densities. The drift surveys have a typical sensitivity of $\sim 0.7 \mathrm{mJy}$ for slow pulsars, but this degrades somewhat for observations far from $\delta=18^{\circ}$ because of the zenith angle dependence of the gain and system temperature. The shorter integration times used in the SHL and doublespeed portion of the CBHL surveys result in sensitivities of 1.5 and $1.0 \mathrm{mJy}$, respectively.

The AC, SHL, DHL, and CBHL surveys cover $4 \%$ of the total sky available at Arecibo, and they significantly increase the area of Arecibo sky which has been surveyed with sensi-

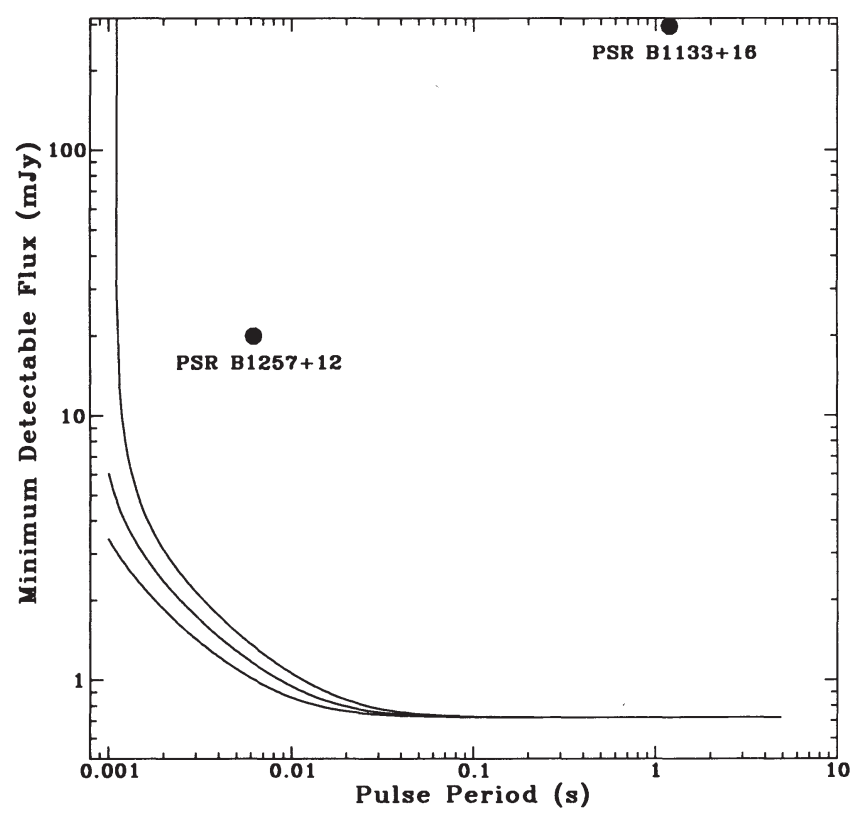

Fig. 4.-The flux limit as a function of period for a typical drift survey from Arecibo using the $2 \times 32$ channel filter bank, with a sample time of $250 \mu$ s and an integration time of $32 \mathrm{~s}$. The curves from bottom to top are for DMs of 10, 20 , and $30 \mathrm{pc} \mathrm{cm}^{-3}$. An intrinsic duty cycle of $6 \%$ is assumed. For other surveys using the filter bank, the sensitivity scales as the square root of the integration time.

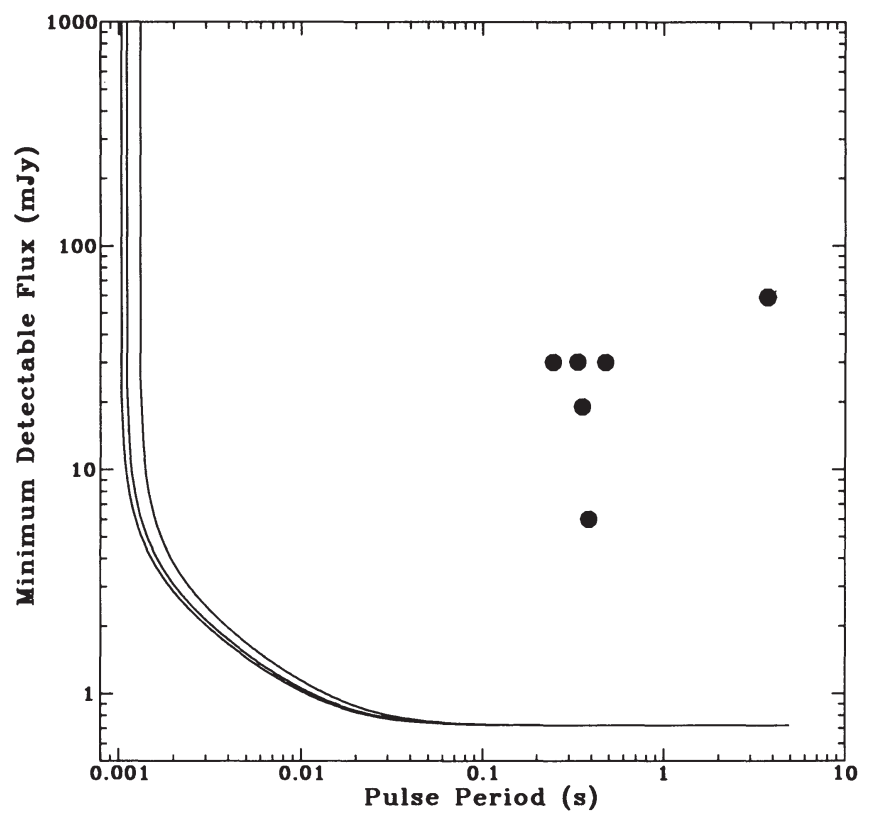

FIG. 5.-The flux limit as a function of period for a typical drift survey from Arecibo using the $2 \times 128$ channel autocorrelator, with a sample time of $506.625 \mu \mathrm{s}$ and an integration time of $32 \mathrm{~s}$. The curves from bottom to top are for DMs of 10,50 , and $100 \mathrm{pc} \mathrm{cm}^{-3}$. An intrinsic duty cycle of $6 \%$ is assumed. For other surveys using the autocorrelator, the sensitivity scales as the square root of the integration time.

tivity to millisecond pulsars. Table 3 summarizes other highlatitude surveys at Arecibo, all of which have sensitivity comparable to the surveys described here. In $\sim 2700$ square degrees, nine recycled pulsars, and 33 normal pulsars were discovered.

The nondetection of millisecond pulsars in the surveys reported in this paper is consistent with the results of other surveys. The previous drift surveys at Arecibo, with $1 \mathrm{mJy}$ sensitivity for slow pulsars (see Table 3), have found millisecond pulsars at an average rate of one per 250 square degrees. The Parkes southern sky survey, with 3 mJy sensitivity to slow pulsars, has discovered millisecond pulsars at a rate of one per 1500 square degrees thus far (Bailes et al. 1994). These rates are consistent with an isotropic distribution of millisecond pulsars, as pointed out by Camilo (1994b). Most of the area covered by surveys in this paper was accounted for by the SHL survey with sensitivity of $\sim 2 \mathrm{mJy}$. Based on area covered and flux limits of these surveys, the expected number of milli-

TABLE 3

\begin{tabular}{|c|c|c|c|c|c|c|}
\hline \multirow{2}{*}{$\begin{array}{c}\text { ArEA } \\
\text { (sq. deg.) }\end{array}$} & \multirow[b]{2}{*}{ TYPE } & \multicolumn{2}{|c|}{$N_{\text {recycled }}$} & \multicolumn{2}{|c|}{$N_{\text {normal }}$} & \multirow[b]{2}{*}{ REFERENCES } \\
\hline & & New & Total & New & Total & \\
\hline $170 \ldots \ldots$ & Drift & 1 & 1 & 3 & 4 & 1 \\
\hline $292 \ldots \ldots$ & Drift & 3 & 3 & 4 & 4 & 2,3 \\
\hline $150 \ldots \ldots$ & Drift & 2 & 2 & 0 & 0 & 4,5 \\
\hline $678 \ldots \ldots$ & Drift & 2 & 2 & 13 & 16 & 6 \\
\hline$\sim 900 \ldots \ldots$ & Drift & 1 & 2 & 13 & 21 & 7,8 \\
\hline $515 \ldots \ldots$ & Various & 0 & 1 & 0 & 7 & 9 \\
\hline
\end{tabular}

Recent High-Latitude Surveys at Arecibo

REFERENCES.-(1) Nice, Taylor, \& Fruchter 1993; (2) Camilo, Nice, \& Taylor 1993; (3) Camilo 1994a; (4) Wolszczan \& Frail 1992; (5) Wolszczan 1991 ; (6) Foster, Wolszczan, \& Cadwell 1994; (7) Ray et al. 1995; (8) Thorsett et al. $1993 ;(9)$ this work. 
second pulsars in the 515 square degrees is between 1 and 2 , whereas we report no new discoveries, and one redetection of PSR B1257+ 12 .

These surveys achieve a nearly constant sensitivity of $\lesssim 1$ mJy over the entire period range of normal pulsars. With their large sky coverage, they place limits on the population of lowluminosity pulsars. The pulsar luminosity distribution is a power law with slope -1 over most of its range, but appears to flatten at $\sim 10 \mathrm{mJy} \mathrm{kpc}^{2}$ (Lyne, Manchester, \& Taylor 1985); however, it still diverges unless a low-luminosity cutoff is imposed. Observationally, the luminosity distribution is very poorly determined below $1 \mathrm{mJy} \mathrm{kpc}^{2}$ (Dewey et al. 1985). The surveys reported in this paper were sensitive to pulsars of luminosity $0.5 \mathrm{mJy} \mathrm{kpc}{ }^{2}$ in a volume of $\sim 2 \times 10^{7} \mathrm{pc}^{3}$, but none were discovered. This implies that the luminosity distribution
$[\Phi(L)]$ as described in Lyne et al. (1985) at least remains flat and possibly begins to turn down by $0.5 \mathrm{mJy} \mathrm{kpc^{2 }}$. A detailed statistical analysis combining the surveys presented in Table 3 and current surveys at Arecibo, Parkes and Jodrell Bank will determine the low end of the pulsar luminosity function and will allow the total number of normal pulsars in the Galaxy and their birthrate to be determined accurately.

We would like to thank José Navarro as well as the staff of Arecibo Observatory for help in performing the observations. The Arecibo Observatory is part of the National Astronomy and Ionosphere Center, which is operated by Cornell University under cooperative agreement with the National Science Foundation.

\section{REFERENCES}

Bailes, M., et al. 1994, ApJ, preprint

Biggs, J. D., \& Lyne, A. G. 1992, MNRAS, 254, 257

Camilo, F. 1994a, personal communication .1994b, in The Lives of the Neutron Stars, ed. A. Alpar, J. van Paradis, \& U. Kiziloglu (Dordrecht: Kluwer), 243

Camilo, F., Nice, D. J., \& Taylor, J. H. 1993, ApJ, 412, L37

Damashek, M., Taylor, J. H., \& Hulse, R. A. 1978, ApJ, 225, L31

Deich, W. T. S. 1994, Ph.D. thesis, California Institute of Technology

Dewey, R. J. 1984, Ph.D. thesis, Princeton Univ.

Dewey, R. J., Taylor, J. H., Weisberg, J. M., \& Stokes, G. H. 1985, ApJ, 294, L25

Foster, R. S., Wolszczan, A., \& Cadwell, B. J. 1994, preprint

Foster, R. S., Wolszczan, A., \& Camilo, F. 1993, ApJ, 410, L91

Hulse, R. A., \& Taylor, J. H. 1974, ApJ, 191, L59

Lyne, A. G., Manchester, R. N., \& Taylor, J. H. 1985, MNRAS, 213, 613

National Astronomy and Ionosphere Center, Cornell University. 1989, Arecibo Observatory User's Manual (NAIC)
Nice, D. J. 1992, Ph.D. thesis, Princton Univ.

Nice, D. J., Taylor, J. H., \& Fruchter, A. S. 1993, ApJ, 402, L49

Phinney, E. S., \& Kulkarni, S. R. 1994, ARA\&A, in press

Ray, P. S., Thorsett, S. E., Jenet, F. A., van Kerkwijk, M. H., Kulkarni, S. R., \& Prince, T. A. 1995, in preparation

Srinivasan, G., \& Bhattacharya, D. 1989, Current Sci., 58, 953

Stokes, G. H., Segelstein, D. J., Taylor, J. H., \& Dewey, R. J. 1986, ApJ, 311, 694

Taylor, J. H., \& Cordes, J. M. 1993, ApJ, 411, 674

Thorsett, S. E., Deich, W. T. S., Kulkarni, S. R., Navarro, J. N., \& Vasisht, G. 1993, ApJ, 416, 182

Wolszczan, A. 1990, IAU Circ., No. 5073

. 1991, Nature, 350, 688

Wolszczan, A., \& Frail, D. A. 1992, Nature, 355, 145 